\title{
Acute coronary artery obstruction after aortic valve replacement surgery and role of transesophageal echocardiography
}

Received May 25, 2017

Revised 1st, July 18, 2017

2nd, August 16, 2017

3rd, August 16, 2017

Accepted August 16, 2017

\section{Corresponding author}

Myung-Soo Jang, M.D., Ph.D.

Department of Anesthesiology and

Pain Medicine, Kyung Hee University

Hospital, 23, Kyungheedae-ro,

Dongdaemun-gu, Seoul 02447,

Korea

Tel: 82-2-958-8589

Fax: 82-2-958-8580

E-mail: haroo82@hanmail.net

\section{Myung-Soo Jang, Jeong-Hyun Choi, Jin-Hee Han, Young In Choi, Jae-Min Kim*, and Hyo Chul Youn ${ }^{+}$}

Department of Anesthesiology and Pain Medicine, *Cardiovascular Division, Department of Internal Medicine, ${ }^{\dagger}$ Department of Thoracic and Cardiovascular Surgery, Kyung Hee University College of Medicine, Seoul, Korea

Acute coronary artery obstruction is a rare but fatal complication of surgical aortic valve replacement (AVR). Sudden coronary spasm, embolization of calcium plaque, and surgical factors can induce acute coronary syndrome after AVR. Here, we report a case of left main coronary ostial obstruction that occurred immediately after weaning from cardiopulmonary bypass. This case highlights the importance of coronary ostial flow patency and assessment of regional wall motion abnormalities with intraoperative transesophageal echocardiography in AVR.

Key Words: Aortic valve replacement, Echocardiography.
Acute coronary artery obstruction is a rare but potentially fatal complication of aortic valve replacement (AVR) surgery $[1,2]$. This adverse event can be induced by sudden coronary spasm, embolization of calcium plaque on the coronary arterial walls or aortic root, or intraoperative iatrogenic events [3]. We present the near-fatal case of a patient who experienced a significant left main coronary ostial obstruction immediately after weaning from cardiopulmonary bypass (CPB). In the case presented here, early detection of regional wall motion abnormalities (RWMAs) in the left coronary arterial territory using intraoperative transesophageal echocardiography (TEE) prevented delayed diagnosis and poor outcome.

\section{CASE REPORT}

A 60-year-old woman was referred to our hospital with dyspnea. The patient had a history of stable angina with percutaneous coronary intervention (PCI) and stenting of the right coronary artery (RCA) four years prior. Preoperative coronary angiography (CAG) revealed a patent RCA stent and moderate stenosis at the proximal left anterior descending coronary artery with an intact coronary ostium. Preoperative TEE revealed preserved biventricular function with intact left coronary ostial flow and severe aortic stenosis with a peak velocity of $7.1 \mathrm{~m} / \mathrm{s}$, mean pressure gradient of $126 \mathrm{mmHg}$, aortic valve area of $0.4 \mathrm{~cm}^{2}$, and heavy calcification of a bicuspid aortic valve (Fig. 1). The patient's coronary risk factors included dyslipidemia and a history of heavy smoking.

Simple AVR was performed through median sternotomy. CPB was initiated, followed by direct aortic root cannulation. Myocardial protection was maintained by intermittent antegrade administration of a cold cardioplegic solution. A mechanical prosthetic aortic valve (St. Jude medical regent mechanical heart valve $19 \mathrm{~mm}$, USA) was implanted, and the aortotomy site was repaired. The operation lasted approximately $5 \mathrm{~h}$, and the total CPB time was $157 \mathrm{~min}$. During surgery, the total injection volumes of crystalloid and colloid were 1,500 and 4,100 $\mathrm{ml}$, respectively. There was a total esti-

This is an Open Access article distributed under the terms of the Creative Commons Attribution Non-Commercial License (http://creativecommons.org/licenses/by-nc/4.0) which permits unrestricted non-commercial use, distribution, and reproduction in any medium, provided the original work is properly cited. 
mated blood loss of more than $1,500 \mathrm{ml}$, for which we administered three units of packed red blood cells and five units of fresh frozen plasma, with apheresis of 1 unit.

Following surgery, smooth weaning from the CPB was performed, no pathologic leakage was identified on TEE, and there was normal valvular motion. However, just before closing the sternotomy site, the patient developed sudden hemodynamic instability, with a systolic blood pressure of $70 \mathrm{mmHg}$ that continued to rapidly decrease. Electrocardiography (ECG) demonstrated myocardial ischemia with ST segment ( $\geq 2 \mathrm{~mm}$ ) change, and the heart rate gradually decreased to less than 40 beats/min followed by progression to ventricular fibrillation and cardiac arrest. At that time, the patient's cerebral oxygen saturation and bispectral index dropped to single-digit values. Cardiopulmonary resuscita-

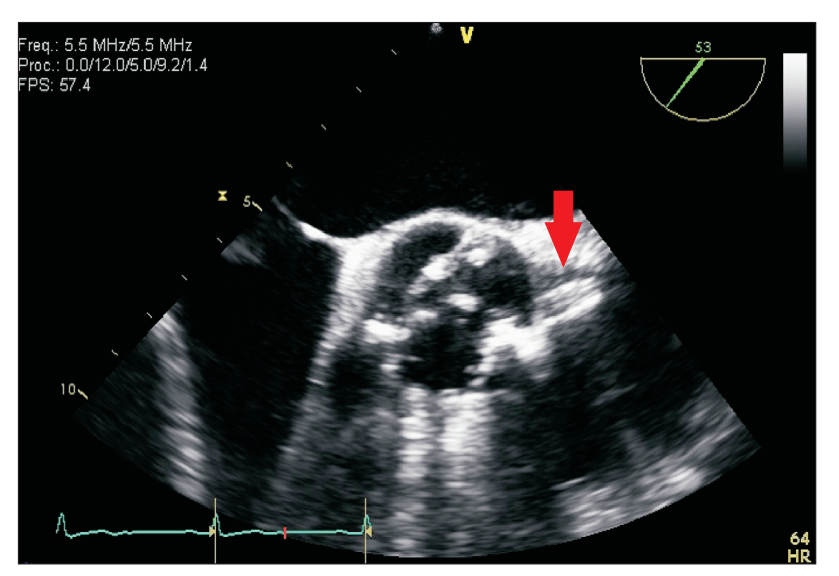

Fig. 1. Preoperative transesophageal echocardiography (TEE) revealed intact left coronary ostium (arrow) and severe aortic stenosis with heavy calcification of the bicuspid aortic valve. tion was started immediately with open cardiac massage and repeated direct defibrillation with 10 joules. With continuous intravenous infusion of $10 \mu \mathrm{g} / \mathrm{kg} / \mathrm{min}$ dobutamine and 0.6 $\mu \mathrm{g} / \mathrm{kg} / \mathrm{min}$ norepinephrine, four $1 \mathrm{mg}$ doses of epinephrine were injected, followed by continuous infusion at $0.1 \mu \mathrm{g} / \mathrm{kg} /$ min. Sinus rhythm with an average heart rate of 90 beats $/ \mathrm{min}$ and mean arterial pressure of $70 \mathrm{mmHg}$ were maintained after resuscitation.

Following resuscitation, we detected a new RWMA on TEE, observed at the base of the septal wall to the apex of the left ventricle. Although we suspected a left coronary perfusion problem, it was difficult to locate the exact coronary lesion (or lesions); thus, we couldn't decide to perform immediate coronary artery bypass graft (CABG) surgery. Veno-arterial extracorporeal membrane oxygenation (ECMO) catheters were cannulated via femoro-femoral access to relieve the burden on the heart. The patient was promptly transferred to the CAG room after the surgeon inserted a polyurethane sponge in the mediastinum between the sternal edges to prevent ventricular rupture. While maintaining a mean arterial pressure of 60-70 $\mathrm{mmHg}$, CAG revealed severe left main coronary ostial stenosis (Fig. 2A).

The cardiologists attributed the coronary compromise to two potential causes, namely, floating calcium debris from AV calcium plaque that had abruptly obstructed the left main coronary ostium intraoperatively and tightening of the left coronary ostium due to external pressure. PCI was deemed too hazardous due to the excessively rigid left main ostia and risk of second cardiac arrest. Indeed, the residual stenosis of the left coronary ostium was $79 \%$ in spite of full balloon
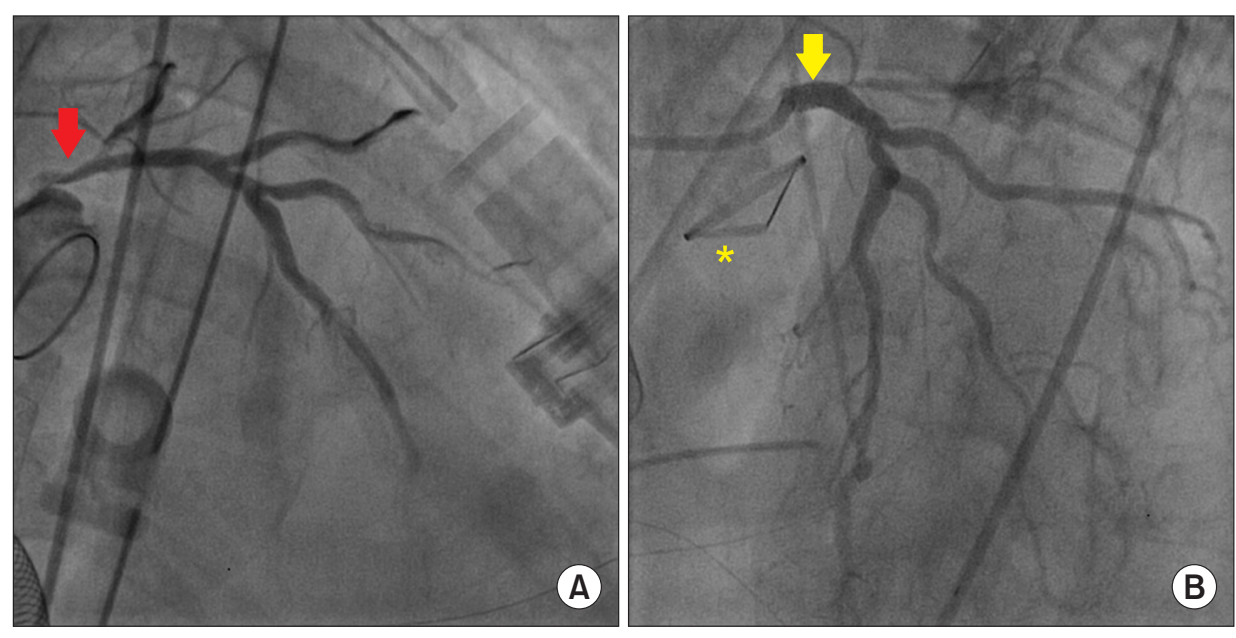

Fig. 2. (A) On coronary angiography, the arrow indicates severe left main coronary ostial stenosis resembled a reversed bird beak. (B) On coronary angiography, the arrow indicates successful stent insertion at the left main coronary ostium. A asterisk denotes the new prosthetic aortic valve. 
expansion. However, a drug-eluting stent (Promus Premier stent $4.0 \times 16 \mathrm{~mm}$, USA) was placed at the left main coronary artery with preparation for immediate CABG operation in case of PCI failure. Fortunately, PCI was successful, and left coronary ostial flow recovered (Fig. 2B).

Following PCI, the patient's blood pressure was 140/100 mmHg with a heart rate of 80 beats/min, and peripheral capillary oxygen saturation $\left(\mathrm{SpO}_{2}\right)$ greater than $98 \%$ was maintained with $10 \mu \mathrm{g} / \mathrm{kg} / \mathrm{min}$ of dobutamine, $0.2 \mu \mathrm{g} / \mathrm{kg} /$ min of norepinephrine, $0.02 \mu \mathrm{g} / \mathrm{kg} / \mathrm{min}$ of epinephrine, and $0.5 \mu \mathrm{g} / \mathrm{kg} / \mathrm{min}$ of nitroglycerine infusion under ECMO. The surgical site was then closed in the operating room. The patient regained consciousness, and the ECMO catheters were removed three days postoperative. On the fourth day, aspirin and warfarin were prescribed; clopidogrel was added two days later with close monitoring of the international normalized ratio. One week after surgery, follow-up echocardiography revealed mild hypokinesia in the left coronary artery territory with preserved left ventricular systolic function and an ejection fraction of $54 \%$ (from $53 \%$ preoperative). After sufficient recovery, the patient was discharged with no other complications.

\section{DISCUSSION}

There have been anecdotal reports of sudden intraoperative coronary ostial obstruction in AVR; so, the incidence rate and mechanism of this complication are unknown $[4,5]$. There are several potential risk factors of acute coronary ostial obstruction in AVR [4,6]. First, intraoperative sympathetic catecholamine release increases cardiac oxygen consumption, which can lead to coronary artery spasm that can be a factor in abrupt hemodynamic collapse. Second, debris embolization from dense calcium buildup in plaques on coronary arterial walls and/or an aortic valve can cause acute coronary obstruction $[5,7]$. Consistent with this possibility, Ono et al. [6] reported a case of left coronary ostial obstruction that occurred approximately 20 minutes after withdrawing $\mathrm{CPB}$. In their case, the suggested mechanism of obstruction was a floating calcified plaque that obstructed the coronary ostium during decalcification of AV lesions. A third potential risk factor for acute coronary ostial obstruction in AVR is occlusion of the left main coronary ostium due to the surgical procedure itself, which can occur with improper positioning of the prosthesis. Indeed, Pillai et al. [3] reported that direct post-traumatic thrombosis due to excessive aortic retractor traction or over-tightening of the new aortic valve suture ring can lead to abrupt intraoperative hemodynamic collapse and fatal outcomes. Likewise, Farid et al. [8] conducted a postmortem autopsy analysis and identified a number of hospital deaths attributed to coronary ostial obstruction after AVR. Specifically, they reported that the prevalence of coronary ostial obstruction following AVR is at least $0.15 \%$. In their study, the authors suggested that patients' underlying conditions and aortic root size could influence the risk of coronary artery compromise; however, they very importantly noted that the cause of death in these patients was attributable, at least in part, to encroachment of the coronary ostia due to surgical factors such as a high level AV suture line.

We considered two potential causes of the complication in the present case. First, hard calcium debris from an AV calcium plaque might have obstructed the coronary ostium. Consistent with this possibility, acute left heart failure induced by calcium debris can occur any time after CPB [6]. A second potential cause of the acute coronary syndrome experienced by the patient was persistent external pressure to overtightening of the coronary ostium. The cardiologists who participated in this case were unable to rule out the latter possibility because the image of the severely obstructed left main coronary ostium resembled a reverse bird beak pattern on CAG.

Intraoperative TEE is a useful tool for identifying the mechanisms of hemodynamically urgent situations in cardiac surgery. Indeed, TEE can provide pivotal information during cardiac valve replacement surgery, including prosthetic valve motion, pathological leaks, and findings related to other valvular diseases. On the other hand, it is easy to overlook adjacent structural problems with TEE. Cardiologists or skilled cardiothoracic anesthesiologists typically focus on prosthetic valvular leaflet motion and paravalvular leakage, which can indicate that the AV is well sutured or well positioned. However, it is much more important with TEE to closely evaluate the new relationship between the bilateral coronary ostium and new prosthetic valve, as well as to monitor for new RWMAs. We know that the coronary arteries run near the valves, and the coronary ostium is located near the aortic valve. Nevertheless, we did not perform a careful evaluation of coronary ostial flow after AVR and overlooked a new RWMA with TEE early during the intraoperative period. 
In cases of intraoperative new-onset acute coronary syndrome, surgeons might find it difficult to identify problematic territories in the surgical field. In such cases, cardiologists and cardiothoracic anesthesiologists can help identify damaged vascular lesions using TEE. Specifically, TEE is the best tool for assessing sudden hemodynamic changes that are of ischemic origin, because RWMA is the first sign prior to ECG changes in such cases [9]. In the present case, detection of the RWMA by TEE was critical for deciding to perform CAG, and doing so prevented a poor outcome due to abrupt coronary arterial obstruction. Usually, a quick return to CPB and urgent $\mathrm{CABG}$ are the ideal solution when an unstable situation is predicted to persist [10]. However, in our case, decannulation had already been performed, and it was difficult to localize the exact location of the coronary lesions.

There is one case report similar to that presented here [4]. In the previous case report, the authors stated that intraoperative verification of coronary patency with TEE or early CAG should be available and ready to perform, because these tools can identify the exact site of injury and mechanism of ischemia. However, the patient described in that case report died following prompt intraoperative RCA bypass surgery without CAG, and the results of a postmortem autopsy identified bilateral obstruction due to surgical factors.

Although CABG has been the choice of treatment for left main disease for many years, PCI and stenting might be equally effective in certain cases due to the continuous improvement and refinement of these techniques over the past 20 years [11]. Thus, coronary obstruction induced by calcium debris is not a contraindication for PCI, despite the associated risk of certain complications $[11,12]$.

In conclusion, if sudden hemodynamic collapse with left heart failure occurs during any point of the intraoperative period following AVR, acute coronary ostial obstruction should be considered. Surgeons should be highly aware of this serious complication after AVR, and anesthesiologists skilled at TEE should work with surgeons to facilitate rapid identification of the etiology of potential coronary complications. Through vigilant awareness and appropriate intervention, it is possible to avoid fatal compromise from acute coronary ostial obstruction, which can ultimately reduce patient mortality.

\section{REFERENCES}

1. Yates JD, Kirsh MM, Sodeman TM, Walton JA Jr, Brymer JF. Coronary ostial stenosis, a complication of aortic valve replacement. Circulation 1974; 49: 530-4

2. Björk V, Henze A, Szamosi A. Coronary ostial stenosis: a complication of aortic valve replacement of coronary perfusion. Scand J Thorac Cardiovasc Surg 1976; 10: 1-6.

3. Pillai JB, Pillay TM, Ahmad J. Coronary ostial stenosis after aortic valve replacement, revisited. Ann Thorac Surg 2004; 78: 2169-71.

4. Turillazzi E, Di Giammarco G, Neri M, Bello S, Riezzo I, Fineschi V. Coronary ostia obstruction after replacement of aortic valve prosthesis. Diagn Pathol 2011; 6: 72.

5. Umran S, Chetty G, Sarkar PK. Acute right coronary ostial stenosis during aortic valve replacement. Int J Prev Med 2012; 3: 295-7.

6. Ono N, Sawai T, Ishii H. Coronary ostial stenosis detected by transesophageal echocardiography after aortic valve replacement: a case report. JA Clin Rep 2017; 3: 14.

7. Yates JD, Kirsh MM, Sodeman TM, Walton JA Jr, Brymer JF. Coronary ostial stenosis, a complication of aortic valve replacement. Circulation 1974; 49: 530-4.

8. Farid S, Page A, Howell N, Goddard M, Abu-Omar Y, Jenkins DP, et al. Coronary ostial compromise in aortic valve replacement: an avoidable complication. Asian Cardiovasc Thorac Ann 2015; 23: 535-42.

9. Nesto RW, Kowalchuk GJ. The ischemic cascade: temporal sequence of hemodynamic, electrocardiographic and symptomatic expressions of ischemia. Am J Cardiol 1987; 59: C23-30.

10. Pifarré R, Grieco J, Sullivan HJ, Scanlon PJ, Johnson SA, Gunnar RM. Coronary embolism: surgical management. Ann Thorac Surg 1980; 30: 564-8.

11. Fajadet J, Chieffo A. Current management of left main coronary artery disease. Eur Heart J 2012; 33: 36-50b.

12. Bangalore S, Vlachos HA, Selzer F, Wilensky RL, Kip KE, Williams DO, et al. Percutaneous coronary intervention of moderate to severe calcified coronary lesions: insights from the National Heart, Lung, and Blood Institute Dynamic Registry. Catheter Cardiovasc Interv 2011; 77: 22-8. 